\title{
ON $\hat{\alpha g}$ COMPACT SPACES \\ ${ }^{1}$ V.Senthilkumaran, ${ }^{2}$ R.Krishnakumar, ${ }^{3}$ Y.Palaniappan* \\ ${ }^{1}$ Associate Professor of Mathematics, Arignar Anna Government Arts College, Musiri, Tamilnadu, India \\ ${ }^{2}$ Assistant Professor of Mathematics,UrumuDhanalakshmi College Kattur, Trichy, Tmilnadu, India \\ ${ }^{3}$ Associate Professor of Mathematics(Retd), Arignar Anna Government Arts College, Musiri, Tamilnadu, India \\ ${ }^{*}$ Corresponding author
}

Keywords: $\hat{\alpha} \mathrm{g}$ compact sets, $\hat{\alpha} \mathrm{g}$ compact spaces, $\hat{\alpha} \mathrm{g}$ open sets.

\begin{abstract}
We introduce and study some subsets of a topological space X called $\hat{a} \mathrm{~g}$ compact sets; $\hat{a} \mathrm{~g}$ compact spaces are defined and their properties are studied.

\section{INTRODUCTION}

Throughout the paper $(X, \tau)$ denotes a topological space in which no separation axiom is assumed. $(X, \tau)$ will be simply denoted by X. Levine [3] initiated semi open sets in topological spaces. Several spaces are defined in terms of semi open sets such as $\mathrm{S}$ - Compact [5], $\mathrm{S}$ - closed space [10], s-closed space [2] etc. In section 3 of the present work, we introduce the notion of $\alpha \mathrm{g}$ compact spaces and study their properties.
\end{abstract}

\section{PRELIMINARIES}

Definition 2.1: A subset $A$ of a topological space $X$ is said to be 1.pre open [4] if $A \subset$ int $\mathrm{cl} A$ and pre closed, if $\mathrm{cl}$ int $\mathrm{A} \subset \mathrm{A}$.

2.regular open [4] if $\mathrm{A}=$ int $\mathrm{cl} \mathrm{A}$ and regular closed if $\mathrm{A}=\mathrm{cl}$ int $\mathrm{A}$.

3.semi open [4] if $\mathrm{A} \subset \mathrm{cl}$ int $\mathrm{A}$ and semi closed if int $\mathrm{cl} A \subset \mathrm{A}$.

Definition 2.2: A subset $A$ of a topological space $X$ is said to $\hat{a} \mathrm{~g}$ closed if int $\mathrm{cl}$ int $\mathrm{A} \subset \mathrm{U}$ whenever $A \subset U$ and $U$ is open in $X$.

The complement of $\hat{a} \mathrm{~g}$ closed set in $\mathrm{X}$ is called $\hat{a} \mathrm{~g}$ open set in $\mathrm{X}$.

The union of all $\hat{a} \mathrm{~g}$ open sets contained in $\mathrm{A}$ is called $\hat{a} \mathrm{~g}$ interior of $\mathrm{A}$ and is denoted by $\hat{a} \mathrm{~g}$ int $\mathrm{A}$. In general $\hat{a} \mathrm{~g}$ int $\mathrm{A}$ is not $\hat{a} \mathrm{~g}$ open.

The intersection of all $\hat{a} \mathrm{~g}$ closed sets containing $\mathrm{A}$ is called the $\hat{a} \mathrm{~g}$ closure of $\mathrm{A}$ and is denoted by $\hat{a} \mathrm{~g} \mathrm{cl} \mathrm{A}$. In general $\hat{a} \mathrm{~g} \mathrm{cl} \mathrm{A}$ is not $\hat{a} \mathrm{~g}$ closed.

In what follows we assume the intersection of $\hat{a} \mathrm{~g}$ closed sets is $\hat{a} \mathrm{~g}$ closed. Then $\hat{a}$ gint $\mathrm{A}$ is $\hat{a} \mathrm{~g}$ open and $\hat{a} \mathrm{~g} \mathrm{cl} \mathrm{A}$ is $\hat{a} \mathrm{~g}$ closed. Also we assume the union of $\hat{a} \mathrm{~g}$ closed sets is $\hat{a} \mathrm{~g}$ closed.

\section{3. $\alpha \hat{g}$ COMPACT SPACES}

Definition 3.1: By a $\hat{a} \mathrm{~g}$ open cover ( or covering ) of a subset A of a topological space $\mathrm{X}$, we mean a collection $\mathbf{C}=\{\mathrm{G} \lambda: \lambda \in \nabla\}$ of $\hat{a} \mathrm{~g}$ open subsets of $\mathrm{X}$ such that $\mathrm{A} \subset \cup\{\mathrm{G} \lambda: \lambda \in \nabla\}$

Definition 3.2: A subset $A$ of a topological space $X$ is said to be $\hat{a} g$ compact if and only if every $\hat{a} \mathrm{~g}$ open cover of A has a finite subcover.

In particular, $\mathrm{X}$ is said to be $\hat{a} \mathrm{~g}$ compact if and only if for every collection $\{\mathrm{G} \lambda: \lambda \in \nabla\}$ of $\hat{a} \mathrm{~g}$ open sets for which $X=\cup\{G \lambda: \lambda \in \nabla\}$ there exists finitely many sets $G \lambda 1, G \lambda 2, \ldots \ldots \ldots G \lambda n$ among the $G \lambda$ such that $X=\cup\{G \lambda i: i=1,2, \ldots n\}$ 
Remark 3.3: Finite union of $\hat{a} \mathrm{~g}$ compact sets is compact.

Definition 3.4: A topological space is said to be $\hat{a} \mathrm{~g}$ Hausdorff if for distinct points $\mathrm{x}, \mathrm{y}$ of $\mathrm{X}$, there exist disjoint $\hat{a} \mathrm{~g}$ open sets $\mathrm{M}$ and $\mathrm{N}$ containing $\mathrm{x}$ and y respectively.

Theorem 3.5: Every $\hat{a} \mathrm{~g}$ compact subset of a $\hat{a} \mathrm{~g}$ Hausdorff space is closed.

Proof: Let A be a $\hat{a} \mathrm{~g}$ compact subset of a $\hat{a} \mathrm{~g}$ Hausdorff space $\mathrm{X}$.

Let us prove $\mathrm{A}^{\prime}$ is $\hat{a} \mathrm{~g}$ open. Let $\mathrm{p} \in \mathrm{A}^{\prime}$.

As $\mathrm{X}$ is $\hat{a} \mathrm{~g}$ Hausdorff, for every $\mathrm{q} \in \mathrm{A}$, there exist disjoint $\alpha \mathrm{g}$ open sets $\mathrm{M}(\mathrm{q})$ and $\mathrm{N}(\mathrm{q})$ of $\mathrm{p}$ and $\mathrm{q}$ respectively.

The collection $\{\mathrm{N}(\mathrm{q}): \mathrm{q} \in \mathrm{A}\}$ is a $\hat{a} \mathrm{~g}$ open cover of $\mathrm{A}$. Since $\mathrm{A}$ is $\hat{a} \mathrm{~g}$ compact, there exist finite number of qi, $\mathrm{s}, \mathrm{i}=1,2, \ldots \ldots$... such that

$A \subset \cup\{N$ qi) $: i=1,2, \ldots n\}$

Let $\mathrm{M}=\bigcap\{\mathrm{M}(\mathrm{qi}): \mathrm{i}=1,2, \ldots . \mathrm{n}\}$

$\mathrm{N}=\cup\{\mathrm{N}(\mathrm{qi}): \mathrm{i}=1,2, \ldots \mathrm{n}\}$

$\mathrm{M}$ is a $\hat{a} \mathrm{~g}$ open set containing $\mathrm{p}$. We have $\mathrm{x} \in \mathrm{N} \Rightarrow \mathrm{x} \in \mathrm{N}(\mathrm{qi})$, for some $\mathrm{i}$

Therefore $\mathrm{x} \notin \mathrm{M}$ (qi) and hence $\mathrm{x} \notin \mathrm{M}$

Hence $\mathrm{M} \cap \mathrm{N}=\phi . \mathrm{A} \subset \mathrm{N}$. So, $\mathrm{M} \cap \mathrm{A}=\phi$

So, $\mathrm{M} \subset \mathrm{A}^{\prime}$. $\mathrm{A}^{\prime}$ contains $\hat{a} \mathrm{~g}$ neighbourhood of each of its points. So, $\mathrm{A}^{\prime}$ is $\hat{a} \mathrm{~g}$ open and hence $\mathrm{A}$ is $\hat{a}$ g closed.

Theorem 3.6: Every $\hat{a} \mathrm{~g}$ closed subset of a compact space is $\hat{a} \mathrm{~g}$ compact.

Proof: Let $\mathrm{F}$ be a $\hat{a} \mathrm{~g}$ closed subset of a $\hat{a} \mathrm{~g}$ compact space $\mathrm{X}$. Let $\mathbf{C}=\{\mathrm{G} \lambda: \lambda \in \nabla\}$ be a $\hat{a} \mathrm{~g}$ open cover of $F$. Then the collection $\mathbf{D}=\{\mathrm{G} \lambda: \lambda \in \nabla\} \cup\{\mathrm{X}-\mathrm{F}\}$ forms $\hat{a} \mathrm{~g}$ open cover of $\mathrm{X}$. Since $\mathrm{X}$ is compact, there is a finite subcollection $\mathbf{D}^{\prime}$ of $\mathbf{D}$ that covers $\mathrm{X}$.

If $\mathrm{X}-\mathrm{F}$ is a member of $\mathbf{D}^{\prime}$, remove it from $\mathbf{D}^{\prime}$. The remaining finite collection will cover F.So $\mathrm{F}$ is $\hat{a} \mathrm{~g}$ compact.

Theorem 3.7: A topological space $\mathrm{X}$ is $\hat{a} \mathrm{~g}$ compact if and only if every collection of $\hat{a} \mathrm{~g}$ closed subsets of $\mathrm{X}$ with finite intersection property (FIP) has a nonempty intersection.

Proof: Let $\mathrm{X}$ be $\hat{a} \mathrm{~g}$ compact. Let $\mathrm{F}=\{\mathrm{F} \lambda: \lambda \in \nabla\}$ be a collection of $\hat{a} \mathrm{~g}$ closed subsets of $\mathrm{X}$ with the FIP .Suppose, $\cap\{F \lambda: \lambda \in \nabla\}=\phi$. Then $\cup\left\{F^{\prime} \lambda: \lambda \in \nabla\right\}=X$, by De Morgan's law.

This means the collection $\left\{\mathrm{F}^{\prime} \lambda: \lambda \in \nabla\right\}$ is a $\hat{a} \mathrm{~g}$ open cover of $\mathrm{X}$. Since $\mathrm{X}$ is compact, we have $\mathrm{X}=\cup\left\{\mathrm{F}^{\prime} \lambda \mathrm{i}: \mathrm{i}=1,2, \ldots \mathrm{n}\right\}$, where $\mathrm{n}$ is finite.

Again by De morgan's law $(\cap\{F \lambda i: i=1,2, \ldots, n\})^{\prime}=X$

This implies $\cap\{F \lambda \mathrm{i}: \mathrm{i}=1, \ldots \mathrm{n}\}=\phi$ contradicting FIP. Hence $\cap\{\mathrm{F} \lambda: \lambda \in \nabla\} \neq \phi$

Conversely, let every collection of $\hat{a} \mathrm{~g}$ closed subsets of X with FIP have nonempty intersection.

Let $\mathbf{C}=\{\mathrm{G} \lambda: \lambda \in \nabla\}$ be a $\hat{a} \mathrm{~g}$ open cover of $\mathrm{X} . \mathrm{X}=\cup\{\mathrm{G} \lambda: \lambda \in \nabla\} . \phi=\bigcap\left\{\mathrm{G}^{\prime} \lambda: \lambda \in \nabla\right\}$.

Thus $\left\{\mathrm{G}^{\prime} \lambda: \lambda \in \nabla\right\}$ is a collection of $\hat{a} \mathrm{~g}$ closed sets with empty intersection. So, this collection does not have the FIP. Hence there exist a finite number of sets $G \lambda i$, $i=1,2, \ldots, n$ such that $\phi=\bigcap\left\{\mathrm{G}^{\prime} \lambda \mathrm{i}: \mathrm{i}=1,2, \ldots, \mathrm{n}\right\}=(\cup\{\mathrm{G} \lambda \mathrm{i}: \mathrm{i}=1,2, \ldots, \mathrm{n}\})^{\prime}$

This implies $X=\cup\{G \lambda i: i=1,2, \ldots, n\})$.

Hence $\mathrm{X}$ is $\hat{a} \mathrm{~g}$ compact.

Theorem 3.8: A topological space $\mathrm{X}$ is $\hat{a} \mathrm{~g}$ compact if and only if every class of $\hat{a} \mathrm{~g}$ closed sets with empty intersection has a finite sub class with empty intersection.

Proof: Let $\mathrm{X}$ be a $\hat{a} \mathrm{~g}$ compact space. Let $\mathbf{C}=\{\mathrm{F} \alpha: \alpha \in \nabla\}$ be a collection of $\hat{a} \mathrm{~g}$ closed subsets of $\mathrm{X}$ such that $\{\mathrm{F} \alpha: \alpha \in \nabla\}=\phi$

Taking complements $\cup\left\{\mathrm{F}^{\prime} \alpha: \alpha \in \nabla\right\}=\mathrm{X}$

The collection $\left\{\mathrm{F}^{\prime} \alpha: \alpha \in \nabla\right\}$ is a $\hat{a} \mathrm{~g}$ open covering of $\mathrm{X}$. As $\mathrm{X}$ is $\hat{a} \mathrm{~g}$ compact, there exist finite number of indices $\alpha 1, \alpha 2, \ldots$. an such that

$\cup\left\{\mathrm{F}^{\prime} \alpha \mathrm{i}: \mathrm{i}=1,2, \ldots \mathrm{n}\right\}=\mathrm{X}$

Taking complements $\cap\{$ F $\alpha i: \mathrm{i}=1,2, \ldots \mathrm{n}\}=\phi$

Conversely, suppose that every class of $\hat{a} \mathrm{~g}$ closed subsets of $\mathrm{X}$ with empty intersection has a finite subclass with empty intersection. Let $C^{*}=\{\mathrm{G} \alpha: \alpha \in \nabla\}$ be an arbitrary $\hat{a} \mathrm{~g}$ open covering of $\mathrm{X}$. 
$\cup\{\mathrm{G} \alpha: \alpha \in \nabla\}=\mathrm{X}$.

Taking complements, $\cap\left\{\mathrm{G}^{\prime} \alpha: \alpha \in \nabla\right\}=. \phi$

The collection $\left\{G^{\prime} \alpha: \alpha \in \nabla\right\}$ has a empty intersection. Hence, there exist finite number of indices $\alpha 1, \alpha 2, \ldots . . \alpha$ such that,

$\cap\left\{\mathrm{G}^{\prime} \alpha \mathrm{i}: \mathrm{i}=1,2, \ldots, \mathrm{n}\right\}=\phi$. Taking complements. $\cup\{\mathrm{G} \alpha \mathrm{i}: \mathrm{i}=1,2, \ldots, \mathrm{n}\}=\mathrm{X}$.

Hence $\mathrm{X}$ is $\hat{a} \mathrm{~g}$ compact.

Definition 3.9: A topological space $\mathrm{X}$ is said to have $\alpha \mathrm{g}$ Bolzano - Weierstrass property ( $\hat{a} \mathrm{~g}$ BWP) if and only if every infinite set in $\mathrm{X}$ has a $\hat{a} \mathrm{~g}$ limit point.

Theorem 3.10: If $\mathrm{A}$ is an infinite subset of a $\hat{a} \mathrm{~g}$ compact space $\mathrm{X}$, then $\mathrm{A}$ has a $\hat{a} \mathrm{~g}$ limit point.

Proof: Let $\mathrm{A}$ have no $\hat{a} \mathrm{~g}$ limit point in $\mathrm{X}$. Then, for every $\mathrm{x} \in \mathrm{X}$, there exists a $\hat{a} \mathrm{~g}$ neighbourhood $\mathrm{Nx}$ of $\mathrm{x}$ which contains no point of A other than (possibly) $\mathrm{x}$. Now the collection $\{\mathrm{Nx}: \mathrm{x} \in \mathrm{X}\}$ forms a $\hat{a} \mathrm{~g}$ open cover of $\mathrm{X}$. Since, $\mathrm{X}$ is $\hat{a} \mathrm{~g}$ compact, there exist finitely many points $\mathrm{x} 1, \mathrm{x} 2, \ldots . . \mathrm{xn}$ in $\mathrm{X}$ such that $\mathrm{X}=\cup\{\mathrm{Nxi}: \mathrm{i}=1,2, \ldots \mathrm{n}\}$

Consequently $\mathrm{A} \subset \cup\{\mathrm{Nxi}: \mathrm{i}=1,2, \ldots \mathrm{n}\}$. As each $\mathrm{Nx}$ contains atmost one point of $\mathrm{A}$, the above relation shows $\mathrm{A}$ has atmost $\mathrm{n}$ points and hence $\mathrm{A}$ is finite, which is a contradiction.

This completes the proof.

Remark 3.11 : No infinite discrete space is $\hat{a} \mathrm{~g}$ compact.

Theorem 3.12 : Let $\mathrm{A}$ and $\mathrm{B}$ be disjoint $\hat{a} \mathrm{~g}$ compact subsets of a $\hat{a} \mathrm{~g}$ Hausdorff space $\mathrm{X}$. Then there exist disjoint $\hat{a} \mathrm{~g}$ open subsets $\mathrm{G}$ and $\mathrm{H}$ such that $\mathrm{A} \subset \mathrm{G}, \mathrm{B} \subset \mathrm{H}$.

Proof: Let $\mathrm{a} \in \mathrm{A}$ be arbitrary but fixed. Let $\mathrm{x} \in \mathrm{B}$ be arbitrary. As $\mathrm{A} \cap \mathrm{B}=\phi, \mathrm{a} \neq \mathrm{x}$.

There exist disjoint $\hat{a} \mathrm{~g}$ open subsets $\mathrm{Gx}$ and $\mathrm{Hx}$ such that $\mathrm{a} \in \mathrm{Gx}, \mathrm{x} \in \mathrm{Hx}$. The collection $\{\mathrm{Hx}: \mathrm{x} \in$ $\mathrm{B}\}$ is a $\hat{a} \mathrm{~g}$ open cover of $\mathrm{B}$. As $\mathrm{B}$ is $\hat{a} \mathrm{~g}$ compact, there exist finitely many points $\mathrm{x} 1, \mathrm{x} 2, \ldots \mathrm{xn} \in \mathrm{B}$ such that $\mathrm{B} \subset \cup\{\mathrm{Hxi}: \mathrm{i}=1,2, \ldots \mathrm{n}\}=\mathrm{Ha}$ (say)

Let $\mathrm{Ga}=\cap\{\mathrm{Gxi}: \mathrm{i}=1,2, \ldots \mathrm{n}\}$. Then $\mathrm{Ga}$ and $\mathrm{Ha}$ are disjoint $\hat{a} \mathrm{~g}$ open sets such that $\mathrm{a} \in \mathrm{Ga}, \mathrm{B} \subset \mathrm{Ha}$..

$\{\mathrm{Ga}: \mathrm{a} \in \mathrm{A}\}$ is a $\hat{a} \mathrm{~g}$ open cover of $\mathrm{A}$. As $\mathrm{A}$ is $\hat{a} \mathrm{~g}$ compact, there exist finitely many points a1,a2, ..... am $\in$ A such that

$\mathrm{A} \subset \cup\{$ Gai $: \mathrm{i}=1,2, \ldots \mathrm{m}\}=\mathrm{G}$ (say).

Let $\mathrm{H}=\cap\{$ Hai $; \mathrm{i}=1,2, \ldots \mathrm{m}\}$. Then $\mathrm{G}$ and $\mathrm{H}$ are disjoint $\hat{a} \mathrm{~g}$ open sets such that $\mathrm{A} \subset \mathrm{G}$ and $\mathrm{B} \subset \mathrm{H}$.

Theorem 3.13 ; Let $\mathrm{X}$ be a $\hat{a} \mathrm{~g}$ connected $\hat{a} \mathrm{~g}$ Hausdorff space[8]. Then no nonempty proper $\hat{a} \mathrm{~g}$ open subset of $\mathrm{X}$ is $\hat{a} \mathrm{~g}$ compact.

Proof $: \mathrm{X}$ is $\hat{a} \mathrm{~g}$ connected. No nonempty $\hat{a} \mathrm{~g}$ open proper subset of $\mathrm{X}$ can be $\hat{a} \mathrm{~g}$ closed. Thus, if $\mathrm{G}$ is a nonempty proper $\hat{a} \mathrm{~g}$ open subset of $\mathrm{X}$, it is not $\hat{a} \mathrm{~g}$ closed. A $\hat{a} \mathrm{~g}$ compact subset of a $\hat{a} \mathrm{~g}$ Hausdroff space is closed. Since $\mathrm{G}$ is not $\hat{a} \mathrm{~g}$ closed, it is not $\hat{a} \mathrm{~g}$ compact.

Theorem 3.14 : If the product of two nonempty spaces is $\hat{a} \mathrm{~g}$ compact, then each factor space is $\hat{a} \mathrm{~g}$ compact.

Proof : Let XxY be the product space of the nonempty spaces $\mathrm{X}$ and $\mathrm{Y}$ and let $\mathrm{XxY}$ be $\hat{a} \mathrm{~g}$ compact.

Then the projection $\pi 1: \mathrm{XxY} \rightarrow \mathrm{X}$ is a $\hat{a} \mathrm{~g}$ irresolute map. Hence $\pi 1(\mathrm{XxY})=\mathrm{X}$ is $\alpha \mathrm{g}$ compact.

For the space $\mathrm{Y}$, the proof is similar.

\section{REFERENCES}

[1].Y.Gnanambal, On gpr continuous function in topological spaces, Indian J. pure Appl. Math 30 (6) (1999) $581-593$.

[2] J.E.Joseph and M.H.Kwach, On s- closed spaces ,Proc.Amer.Math.Soc.80(2):341- 348,1980.

[3] N.Levine, Semi open sets and semi continuity in topological spaces, Amer.Math. Monthly 70 (1963) 36-41.

[4] A.S.Mashhour, M.E.Abd El-Monsef and S.N.ElDeeb, On pre - continuous and weak pre continuous mappings, Proc. Math and Phy.Soc.Egypt 53 (1982) 47- 53. 
[5] S.S.Mohammad, On Semi compact sets and associated properties, Int.J.Math.Math. Sci. Article ID 465387: 1-8,2009.

[6] O.Njastad, On some classes of nearly open sets, Pacific.J.Math 15 (3) (1965) 961-970.

[7] V.Senthilkumaran, R.Krishnakumar and Y.Palaniappan, On $\alpha$ generalized closed sets, Int.J.Math Archive. Feb 2014, 187-191.

[8] V.Senthilkumaran, R.Krishnakumar and Y.Palaniappan, $\alpha \mathrm{g}$ connected spaces ,Bulletin of Society for Mathematical Services\&Standards,vol(3) Issue2(2014) 19-28.

[9] L.A.Steen and J.A.Seebach, Counter examples in topology, Holt, Rinehart and Winston Inc. U.S.A.1970.

[10] T.Thompson, S- closed spaces, Proc.Amer.Math.Soc.60 (61): 335-338, 1976. 